\title{
Probabilistic Mechanics of Quasibrittle Structures: Strength, Lifetime and Size Effect
}

\author{
Zdeněk P BažantandJia-LiangLe* \\ Department of Mechanical Engineering and Material Science and Engineering, USA \\ *Corresponding author: Zdeněk P BažantandJia-LiangLe, Department of Mechanical Engineering and Material Science and Engineering, USA

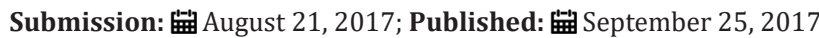

\section{Opinion}

Quasibrittle materials are becoming increasingly important for modern engineering. They include tough (coarse-grained) or toughened ceramics, concretes, rocks (including shale), fiber composites, sea ice, wood, carton, stiff soils, rigid foams, glass, bone, dental and biomaterials, as well as all brittle materials on the micro or nano scale. Their salient feature is that the fracture process zone size is non-negligible compared to the structural dimensions. This causes intricate energetic and statistical size effects and leads to size-dependent probability distribution of strength that is transitional between Gaussian and Weibullian. At the failure probability of 1 in a million, which is the maximum tolerable as it is orders of magnitude smaller than other risks to which people are willingly exposed, the strength difference between the Gaussian and Weibull distributions is enormous-almost 2:1. At this probability level, the strength distribution cannot be tested directly and requires a fundamental theory, whose experimental verification can be obtained through the predicted size effect. The size effect on strength statistics, a quintessential property of quasibrittle materials, has profound implications for reliabilitybased structural design, in which the widely used reliability indices and safety factors need to be modified to incorporate the size dependence.

Probabilistic Mechanics of Quasibrittle Structures presents a recently developed mechanics-based probabilistic theory of strength and lifetime statistics, which aims to address the ensuing difficult challenges for safe design of quasibrittle structures.
Drawing upon years of practical experience, fundamental theoretical advances and laboratory testing, and using numerous examples and illustrative applications, the authors cover:

a. Rigorous theory with detailed derivations yet no superfluous mathematical sophistication.

b. Mathematical relation of quasibrittle strength statistics to the size effect on structural strength.

c. Comprehensive experimental verifications, which include extensive data on strength, lifetime, size effect and fatigue kinetics of engineering and dental ceramics.

d. Realistic approximations for reliability-based structural design.

e. Fracture kinetics under both static and cyclic fatigue loading and its size effect.

f. Statistical multi scale transition bridging theatomistic scale and structural scale.

g. Statistics of structural strength and lifetime, size effect and reliability indices.

h. Ramification to gate dielectrics breakdown with analogous mathematical formulation. 